\title{
Precoding-Aided Bandwidth Optimization for High Throughput Satellite Systems
}

\author{
Tedros Salih Abdu, Lei Lei, Steven Kisseleff, Eva Lagunas, Symeon Chatzinotas and Björn Ottersten \\ Interdisciplinary Centre for Security, Reliability and Trust (SnT), \\ University of Luxembourg, Luxembourg \\ Email: \{tedros-salih.abdu, lei.lei, steven.kisseleff, eva.lagunas, symeon.chatzinotas, bjorn.ottersten\}@uni.lu
}

\begin{abstract}
Linear precoding boosts the spectral efficiency of the satellite system by mitigating the interference signal. Typically, all users are precoded and share the same bandwidth regardless of the user demand. This bandwidth utilization is not efficient since the user demand permanently varies. Hence, demand-aware bandwidth allocation with linear precoding is promising. In this paper, we exploited the synergy of linear precoding and flexible bandwidth allocation for geostationary (GEO) high throughput satellite systems. We formulate an optimization problem with the goal to satisfy the demand by taking into account that multiple precoded user groups can share the different bandwidth chunks. Hence, optimal beam groups are selected with minimum bandwidth requirement to match the per beam demand. The simulation results show that the proposed method of combining bandwidth allocation and linear precoding has better bandwidth efficiency and demand satisfaction than benchmark schemes.

Index Terms-GEO satellite, bandwidth allocation, demand satisfaction, linear precoding.
\end{abstract}

\section{INTRODUCTION}

The number of commercial High Throughput Satellite (HTS) systems has increased over the last decade thanks to the extensive investments made by satellite operators in response to the consumer broadband market. The HTS systems typically offer coverage via narrow spot-beams, and satellite operators must define an appropriate bandwidth-to-beam assignment to avoid co-channel interference. The classical scheme follows a four-color frequency-reuse, where colors refer to a frequency-polarization state considering two frequencies and two polarizations [1], [2]. Hence, we can re-use the same frequency band on non-adjacent beams while using different color frequencies on adjacent beams. The number of color frequencies determines the level of interference occurrence and the bandwidth allocation per beam. The interference is less for a higher number of the color frequency (e.g., 4color scheme) but the lower bandwidth allocation per beam. In contrast, the lower number of color frequencies (e.g., 1color scheme) is the higher bandwidth allocation per beam but the higher the interference occurrence. Therefore, the HTS needs a smart bandwidth allocation with efficient interference

This work has been supported by the Luxembourg National Research Fund (FNR) under the project FlexSAT (C19/IS/13696663) and the Aides à la Formation-Recherche (AFR) Grant INSAT - "Power and Bandwidth Allocation for INterference-Limited SATellite Communication Systems". mitigating/control technique. Consequently, we obtain high spectral efficiency that enables us to match broadband traffic loads [3].

Several interference management techniques have been proposed for HTS in the literature by optimizing the system's frequency and power while matching the per beam demand [4]. The power and/or bandwidth are allocated according to the demand while considering the minimum interference among the users. Analytical power and/or bandwidth optimization to satisfy the per beam demand under acceptable user interference have been considered in [5]-[10]. Similarly, power and/or bandwidth allocation using metaheuristic and machine learning approach to match the per beam demand is proposed in [11]-[14] and [15], [16], respectively. Furthermore, Beam Hopping technique based on scheduling have been studied in [15], [17], [18]. However, the offered capacity of the above mentioned techniques is limited due to the interference [9]. Hence, we cannot satisfy the user demand for high demand requests.

Advanced interference management techniques using linear precoding have been studied in the literature further to increase the system's offered capacity [19]. The linear precoder for satellite has been considered first with the concept of the multiple-input-multiple-output (MIMO) method in [20]. In [21], linear precoding and beamforming have been considered. Later, linear precoding with multicasting and scheduling has been considered in [22]-[24], and [25], respectively. Furthermore, the performance of linear precoding for the hot-spot scenario has been studied in [26]. However, joint precoding and bandwidth allocation with the goal of demand satisfaction has not been considered yet.

In this paper, we exploited the synergy of linear precoding and flexible bandwidth allocation to increase the spectral efficiency, bandwidth utilization, and demand satisfaction of the system. The contribution of the paper is described as follows.

- Firstly, we design a tailored utility function for overloaded and resource-limited HTS systems to jointly minimize requested-offered data mismatch and total bandwidth consumption.

- Secondly, we propose a Precoding-Aided Bandwidth Optimization (PABO) scheme to enable high-performance bandwidth utilization in HTS systems. We obtain the 
bandwidth allocation by formulating and solving an optimization problem for $\mathrm{PABO}$ such that more requested data can be satisfied by using less bandwidth.

- Finally, the performance of PABO is illustrated through numerical results. It is shown that the PABO performs better compared with benchmark schemes in terms of bandwidth utilization and demand satisfaction.

The paper is organized as follows. Section II provides the system model. Section III contains the problem formulation and the proposed solution. The simulation result is presented in Section IV. Finally, the conclusion of the paper is provided in Section V.

Notation: The matrices and vectors are represented by boldface of upper case and lower case letters, respectively. The sum of elements on the main diagonal of the square matrix represented by Trace $\{$.$\} . The transpose of a vector and$ conjugate transpose of a vector represented by [.] $]^{T}$ and $[.]^{H}$, respectively.

\section{SySTEM MODEL}

Consider a downlink multibeam GEO satellite with $N$ beams, as shown in Fig. 1. The satellite is required to match the users' data demands within the total available bandwidth $B_{\text {tot }}$. In each spectrum block, a single beam or a group of beams can be served at the same time. Assuming single user per beam, the maximum number of groups is $M=2^{N}-1$ and the set of clusters (groups) is denoted as $\mathcal{G}=\left\{G_{1}, G_{2}, \ldots, G_{m}, \ldots, G_{M}\right\}$, where $G_{m}$ is the $m$ th group of beams. We denote the $m$ th group channel matrix as $\mathbf{H}_{m}=\left[\mathbf{h}_{m}[1], \mathbf{h}_{m}[2], \ldots, \mathbf{h}_{m}\left[\left|G_{m}\right|\right]\right]^{T}$ with $\mathbf{h}_{m}[i]=$ $\left[h_{i, m}[1], h_{i, m}[2], \ldots, h_{i, m}\left[\left|G_{m}\right|\right]\right]^{T}$, where $\mathbf{h}_{m}[i]$ is the channel vector from the satellite to the user in the $i$ th beam belonging to the $m$ th group and $\left|G_{m}\right|$ is the cardinality of the $m$ th group. Furthermore, the channel coefficient $h_{i, m}[j]$ is given by

$$
h_{i, m}[j]=\frac{\sqrt{G_{R} G_{i}[j]}}{4 \pi \frac{d_{i}}{\lambda}},
$$

where $\lambda$ is the is the wavelength, $G_{R}$ is the user antenna gain, $G_{i}[j]$ denotes the received gain from the $j$ th beam by the $i$ th user and $d_{i}$ is the slant range between the satellite and the $i$ th user.

The corresponding precoding matrix for the $m$ th group is defined as $\mathbf{W}_{m}=\left[\mathbf{w}_{m}[1], \mathbf{w}_{m}[2], \ldots, \mathbf{w}_{m}\left[\left|G_{m}\right|\right]\right]^{T}$ with $\mathbf{w}_{m}[i]=\left[w_{i, m}[1], w_{i, m}[2], \ldots, w_{i, m}\left[\left|G_{m}\right|\right]\right]^{T}$, where $\mathbf{w}_{m}[i]$ is the precoding weight vector for user in the $i$ th beam belonging to the $m$ th group. We obtain $\mathbf{W}_{m}$ based on Minimum Mean Square Error (MMSE) precoding technique [9] as follows

$$
\mathbf{W}_{m}=\eta \hat{\mathbf{W}}_{m}
$$

with

$$
\hat{\mathbf{W}}_{m}=\mathbf{H}_{m}^{H}\left(\mathbf{H}_{m} \mathbf{H}_{m}^{H}+\beta \mathbf{I}\right)^{-1},
$$

$$
\eta=\sqrt{\frac{P_{m}\left|G_{m}\right|}{\operatorname{Trace}\left\{\hat{\mathbf{W}}_{m} \hat{\mathbf{W}}_{m}^{H}\right\}}} .
$$

where $\beta$ is the regularization factor given by $\beta=N_{0} B_{m} / P_{m}$, $N_{0}$ is the noise density in [W/Hz], $B_{m}$ is the bandwidth of the spectrum block assigned to the $m$ th group and $P_{m}$ is the maximum transmit power allowed any user of the $m$ th group. Hence, the signal-to-interference-plus-noise ratio of the $i$ th user in the $m$ th group is given by

$$
\gamma_{m}[i]=\frac{\left|\mathbf{h}_{m}^{T}[i] \mathbf{w}_{m}[i]\right|^{2}}{\sum_{j=1, j \neq i}^{\left|G_{m}\right|}\left|\mathbf{h}_{m}^{T}[i] \mathbf{w}_{m}[j]\right|^{2}+N_{0} B_{m}} .
$$

Then, the Shannon capacity for the $i$ th user in the $m$ th group is

$$
C_{m}[i]=B_{m} \log _{2}\left(1+\gamma_{m}[i]\right) .
$$

Subsequently, the overall offered capacity by the system to the $i$ th user is provided as

$$
C[i]=\sum_{G_{m} \in \mathcal{G}, i \in G_{m}} C_{m}[i] .
$$

Finally, for user demand $D[i]$, the unmet system capacity is given by

$$
C_{\text {unmet }}=\sum_{i=1}^{N} \max (D[i]-C[i], 0) \text {. }
$$

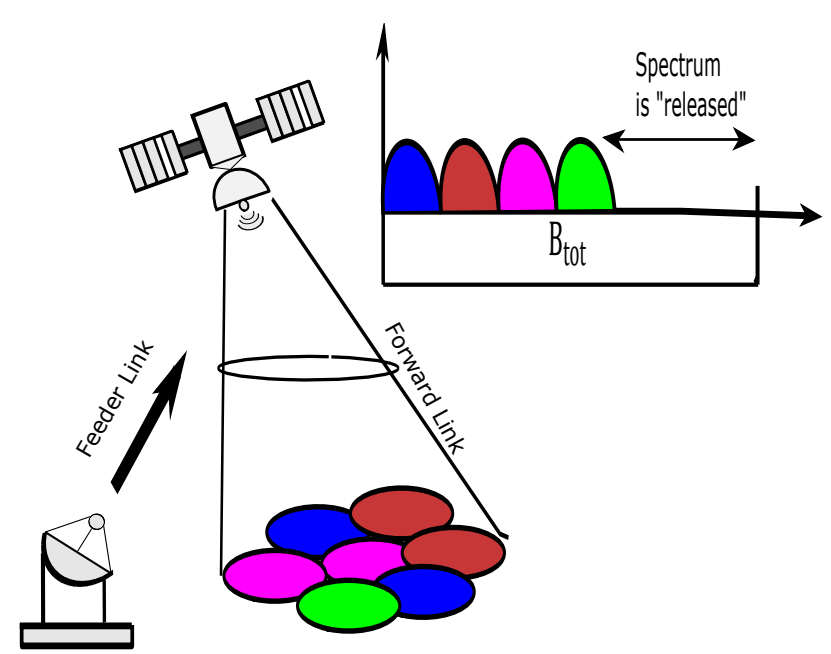

Fig. 1. Example of bandwidth-to-beam assignment.

\section{Problem Formulation and Proposed Solution}

In this work, we combine linear precoding and flexible bandwidth allocation to satisfy the per beam demand while efficiently utilizing the system bandwidth. Hence, according to the demand and user interference, the system allocates a spectrum block bandwidth to groups of users while minimizing bandwidth usage. Accordingly, the users within a group share the same spectrum block to satisfy their respective demands. In this context, we considered a multi-objective optimization, 
where the unmet system capacity and the total bandwidth of the system are minimized. Therefore, we formulate the optimization as follows

$$
\begin{array}{ll}
\underset{B_{m}, P_{m} \forall_{m}}{\operatorname{minimize}} & \sum_{i=1}^{N} \max (D[i]-C[i], 0)+\sum_{G_{m} \in \mathcal{G}} B_{m} \\
\text { s.t. } & T 1: \sum_{G_{m} \in \mathcal{G}} B_{m} \leq B_{\text {tot }}, \\
& T 2: B_{m} \geq 0, \forall_{m}, \\
& T 3: \sum_{G_{m} \in \mathcal{G}}\left|G_{m}\right| P_{m} \leq P_{\text {total }}, \\
& T 4: P_{m} \geq 0, \forall_{m} .
\end{array}
$$

The constraint $T 1$ describes that overall bandwidth utilization should not exceed the total available system bandwidth. The $T 2$ constraint indicates that $B_{m}$ should be non-negative. The constraint $T 3$ describes that the total transmit power of the system should not exceed the total available system power $P_{\text {total }}$. The T4 constraint indicates that $P_{m}$ should be nonnegative.

We observed that the units of the objective function of the problem are not the same. Hence, we normalize the objective function to

$$
f_{\text {multi_obj }}=\sum_{i=1}^{N} \max \left(1-\frac{C[i]}{D[i]}, 0\right)+\sum_{G_{m} \in \mathcal{G}} \frac{B_{m}}{B_{\text {tot }}} .
$$

The unmet system capacity and SINR $\gamma_{m}[i]$ makes (9) nonconvex. We remove the non-differentiability of the unmet system capacity by replacing it with upper bound slack variable $u[i], \forall_{i}$, where $u[i] \geq 0, \forall_{i}$ and $u[i] \geq 1-\frac{C[i]}{D[i]}, \forall_{i}$. Furthermore, to resolve the non-convexity $\gamma_{m}[i]$, we avoid the direct dependency of $\gamma_{m}[i]$ from $B_{m}$ and $P_{m}$. For this, we assume that the power spectral density $S_{\mathrm{psd}}[\mathrm{W} / \mathrm{Hz}]$ is given per beam. Hence, we equivalently express the transmit power $P_{m}$ in terms of $S_{\mathrm{psd}}$ and $B_{m}$, i.e., $P_{m}=S_{\mathrm{psd}} B_{m}$. Then, (5) written as.

$$
\gamma_{m}[i]=\frac{\left|\mathbf{h}_{m}^{T}[i] \widetilde{\mathbf{w}}_{m}[i]\right|^{2}}{\sum_{j=1, j \neq i}^{\left|G_{m}\right|}\left|\mathbf{h}_{m}^{T}[i] \widetilde{\mathbf{w}}_{m}[j]\right|^{2}+N_{0}}
$$

where $\widetilde{\mathbf{w}}_{m}[i]=\frac{\mathbf{w}_{m}[i]}{\sqrt{B_{m}}}$ is the precoding vector in terms of power spectral density ${ }^{1}$. Furthermore, $T 3$ is re-written as

$$
T 3: \sum_{G_{m} \in \mathcal{G}}\left|G_{m}\right| S_{\mathrm{psd}} B_{m} \leq P_{\text {total }} .
$$

Note that the total transmit power constraint $T 3$ is a function of the optimization variable $B_{m}$. Finally, the equivalent problem of (9) written as follows

${ }^{1}$ Note that the precoding matrix in termes of spectral power density is $\tilde{\mathbf{W}}_{m}=\frac{\mathbf{W}_{m}}{\sqrt{B_{m}}}=\widetilde{\eta} \hat{\mathbf{W}}_{m}$ with $\tilde{\eta}=\frac{\eta}{\sqrt{B_{m}}}=\sqrt{\frac{P_{m}\left|G_{m}\right|}{B_{m} \operatorname{Trace}\left\{\hat{\mathbf{W}}_{m} \hat{\mathbf{W}}_{m}^{H}\right\}}}=$ $\sqrt{\frac{S_{\mathrm{psd}}\left|G_{m}\right|}{\operatorname{Trace}\left\{\hat{\mathbf{W}}_{m} \hat{\mathbf{W}}_{m}^{H}\right\}}}$ and $\beta=N_{0} / S_{\mathrm{psd}}$. Hence, $\widetilde{\mathbf{W}}_{m}$ is independent of $B_{m}$ and $P_{m}$.

$$
\underset{B_{m}, \forall_{m}, u[i], \forall_{i}}{\operatorname{minimize}} \quad \sum_{i=1}^{N} u[i]+\sum_{G_{m} \in \mathcal{G}} \frac{B_{m}}{B_{\text {tot }}}
$$

s.t.

$$
\begin{aligned}
& T 1, T 2, T 3 \\
& T 5: 1-\frac{C[i]}{D[i]} \leq u[i], \forall_{i} \\
& T 6: u[i] \geq 0, \forall_{i} .
\end{aligned}
$$

Problem (12) is a linear program that can be solved by well-established solvers [27].

\section{Simulation Results}

In this section, the proposed Precoding-Aided Bandwidth Optimization (PABO) performance is evaluated through simulation. The system parameters used for this simulation are shown in Table I. We consider $N=20$ beams with a single user per beam. The beams shown in Fig. 2 are generated using a Direct Radiating Antenna (DRA), with 750 elements spaced 5 lambda apart and provided by the European Space Agency (ESA). We obtain the simulation results by averaging over $L=100$ Monte-Carlo runs. In each run, the user location is randomly selected from a uniform distribution within the beam coverage. Fig. 2 shows an example of randomly selected users for one time Monte-Carlo run. Additionally, the requested demand is assumed to be equal for all beams, i.e., $D[i]=D, \forall_{i}$, and we vary the value of $D$ between 100 and 1000 Mbps. Note that since the power spectral density per beam is $S_{p s d}=-78.8$ $\mathrm{dBW} / \mathrm{Hz}$, then the total transmit power of the system is given by $P_{\text {total }}=S_{\text {psd }} B_{\text {tot }} L=132 \mathrm{~W}$.

TABLE I

SYSTEM PARAMETERS

\begin{tabular}{|c|c|}
\hline Parameter & Value \\
\hline Satellite Orbit & $13^{\circ} \mathrm{E}$ \\
\hline Satellite Beam Pattern & Provided by ESA \\
\hline Number of beams $(N)$ & 20 \\
\hline System Bandwidth $\left(B_{t o t}\right)$ & $500 \mathrm{MHz}$ \\
\hline Noise power density $\left(N_{0}\right)$ & $-204 \mathrm{dBW} / \mathrm{Hz}$ \\
\hline Max. beam gain $\left(G_{i}[j]\right)$ & $51.8 \mathrm{dBi}$ \\
\hline User antenna gain $\left(G_{R}\right)$ & $39.8 \mathrm{dBi}$ \\
\hline Power spectral density $\left(S_{\text {spd }}\right)$ & $-78.8 \mathrm{dBW} / \mathrm{Hz}$ \\
\hline Total available transmit power $\left(P_{\text {total }}\right)$ & $132 \mathrm{~W}$ \\
\hline
\end{tabular}

We compare the proposed scheme with the following benchmark schemes:

1) 1-Color full bandwidth with precoding scheme (1-Color with precoding):

$$
C[i]=B_{\text {tot }} R[i]
$$

with

$$
R[i]=\log _{2}\left(1+\frac{\left|\mathbf{h}^{T}[i] \widetilde{\mathbf{w}}[i]\right|^{2}}{\sum_{j=1, j \neq i}^{N}\left|\mathbf{h}^{T}[i] \widetilde{\mathbf{w}}[j]\right|^{2}+N_{o}}\right),
$$

where $\mathbf{h}[i] \in \mathbb{C}^{1 \times N}$ is the $i$ th channel vector, and $\mathbf{w}[i] \in$ $\mathbb{C}^{N \times 1}$ is the $i$ th MMSE precoding vector. 


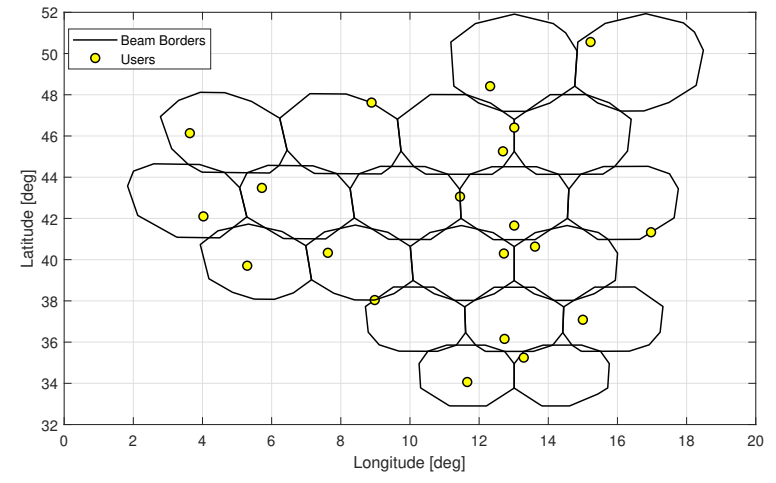

Fig. 2. $N=20$ beam scenario with an example of user distribution

2) 4-Color scheme without precoding (4-Color w/o precoding)

$$
C[i]=B_{c} R[i]
$$

with

$$
\begin{gathered}
R[i]=\log _{2}\left(1+\frac{\left|h_{i}[i]\right|^{2} x_{k}[i] p[i]}{\sum_{j=1, j \neq i}^{N}\left|h_{i}[j]\right|^{2} x_{k}[j] p[j]+N_{0} B_{c}}\right) \\
B_{c}=\frac{B_{\text {tot }}}{4}, \\
p[i]=\frac{P_{\text {total }}}{N}, \forall_{i},
\end{gathered}
$$

where $B_{c}$ is the bandwidth chunk per color, $p[i]$ is the $i$ th transmitted power and $x_{k}[i] \in\{0,1\}, k=1,2,3,4$ with $x_{k}[i]=1$ indicates that the $k$ th color is assigned to the $i$ th beam.

The performance indicators considered for the simulation are the average unmet system capacity and the average utilized bandwidth [8]. Furthermore, the Average Power Consumption (APC) is obtained as

$$
\mathrm{APC}=\frac{1}{L} \sum_{l=1}^{L} \sum_{G_{m} \in \mathcal{G}}\left|G_{m}\right| S_{\mathrm{psd}} B_{m} .
$$

Fig. 3 shows the average unmet system capacity of PABO and benchmark schemes. We observed that all schemes have zero unmet system capacity up to $300 \mathrm{Mbps}$. However, for demand above $300 \mathrm{Mbps}$, the proposed scheme has a lower unmet system capacity than the benchmark schemes. For instance, the unmet system capacity of PABO at $600 \mathrm{Mbps}$ and $800 \mathrm{Mbps}$ is $4 \mathrm{Mbps}$ and $44 \mathrm{Mbps}$, respectively, whereas, for the 1-Color with precoding and 4-Color w/o precoding schemes, the unmet system capacity is $\{13,56\}$ Mbps and $\{141,341\}$ Mbps, respectively. Moreover, the PABO can satisfy the beam demand up to $400 \mathrm{Mbps}$, while the benchmark schemes are limited to $300 \mathrm{Mbps}$ per beam. Hence, the overall performance of PABO is better than the benchmark schemes.

Fig. 4 shows the bandwidth utilization of all schemes. The PABO has lower bandwidth utilization compared with the 1Color with precoding and 4-Color w/o precoding schemes. The benchmark schemes are fully utilized the system bandwidth, whereas the PABO allocates bandwidth according to the demand and the user interference. However, for demand above $700 \mathrm{Mbps}$, the PABO fully utilized the system bandwidth. Hence, the bandwidth utilization of all schemes is the same for higher demand.

Fig. 5 shows the power consumption of the proposed scheme and the benchmark schemes. We obtain using (15) the power consumption by multiplying the power spectral density with the bandwidth. Hence, similar to Fig. 4, the overall power consumption of PABO is lower compare with the benchmark schemes. The power allocation of 1-Color with precoding and 4-Color w/o precoding schemes are the same regardless of the requested demand. However, PABO allocates power according to the demand. Moreover, PABO has advantages in selecting groups that have orthogonal channel characteristics. Consequently, the power consumption is lower than the benchmark schemes.

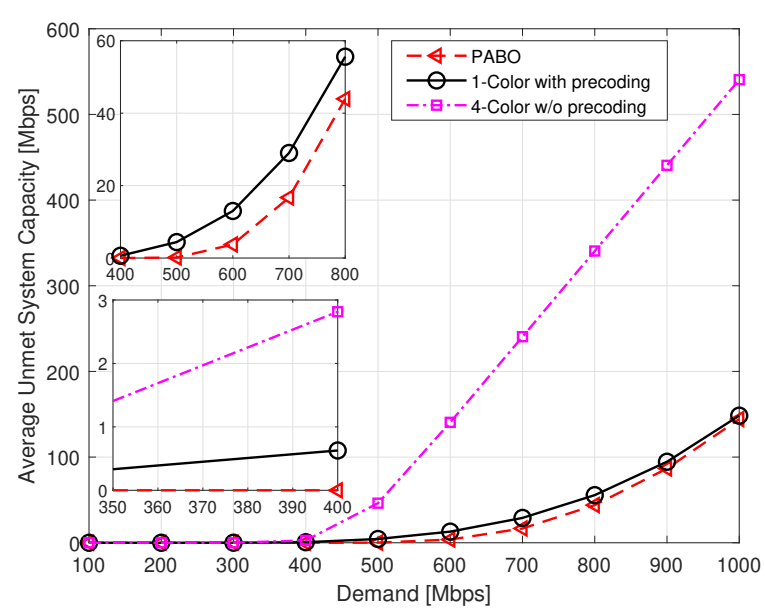

Fig. 3. Unmet system capacity of PABO and benchmark schemes.

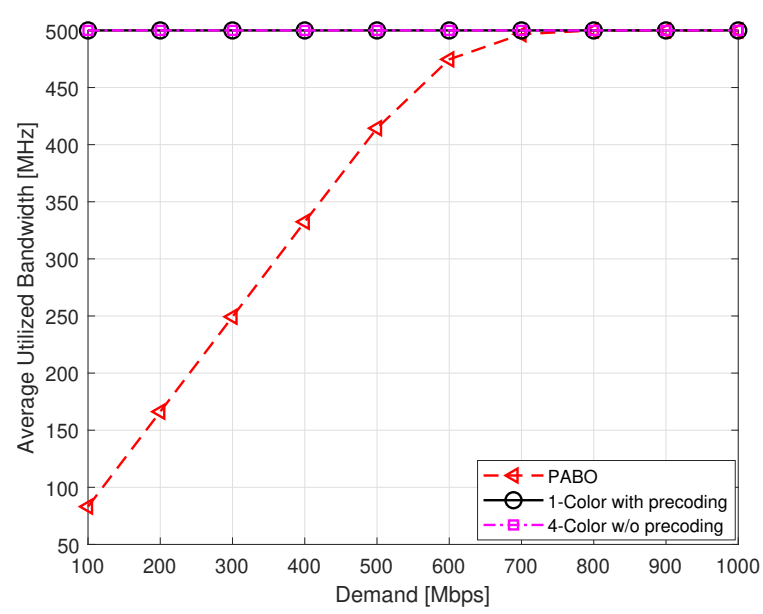

Fig. 4. Utilized bandwidth of PABO and benchmark schemes. 


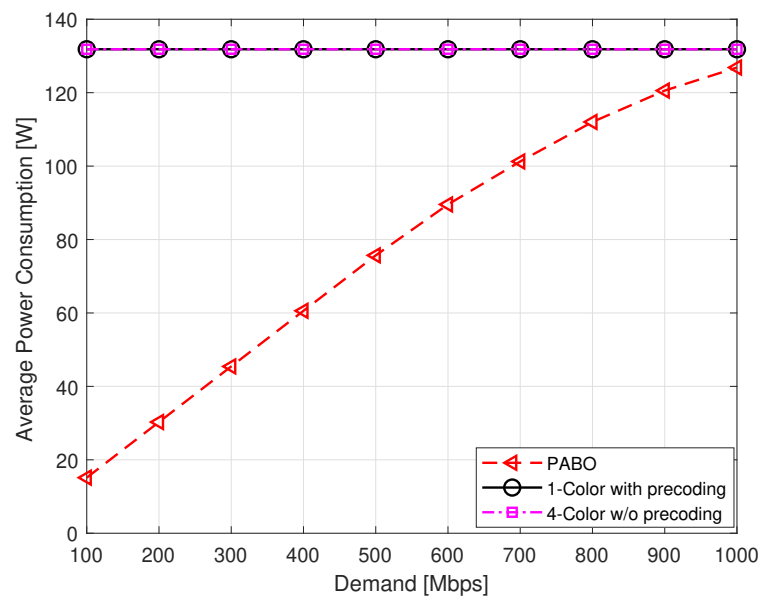

Fig. 5. Power consumption of PABO and benchmark schemes.

\section{Conclusions}

In this paper, we exploited the synergy of linear precoding and flexible bandwidth allocation for GEO high throughput satellite systems. The proposed method determines optimal beam groups that satisfy per beam demand with minimum satellite resource utilization including both on-board amplified bandwidth and transmit power. The simulation result shows that the proposed solution has better bandwidth efficiency and demand satisfaction than benchmark schemes.

\section{REFERENCES}

[1] A. I. Perez-Neira, M. A. Vazquez, M. B. Shankar, S. Maleki, and S. Chatzinotas, "Signal processing for high-throughput satellites: Challenges in new interference-limited scenarios," IEEE Signal Processing Magazine, vol. 36, no. 4, pp. 112-131, 2019.

[2] O.Kodheli et al., "Satellite communications in the new space era: A survey and future challenges," IEEE Communications Surveys Tutorials, vol. 23, no. 1, pp. 70-109, 2021.

[3] M. A. Vazquez, A. Perez-Neira, D. Christopoulos, S. Chatzinotas, B. Ottersten, P. Arapoglou, A. Ginesi, and G. Tarocco, "Precoding in multibeam satellite communications: Present and future challenges," IEEE Wireless Communications, vol. 23, no. 6, pp. 88-95, 2016.

[4] S. Kisseleff, E. Lagunas, T. S. Abdu, S. Chatzinotas, and B. Ottersten, "Radio resource management techniques for multibeam satellite systems," IEEE Communications Letters, pp. 1-1, 2020.

[5] T. S. Abdu, S. Kisseleff, E. Lagunas, and S. Chatzinotas, "Flexible Resource Optimization for GEO Multibeam Satellite Communication System," IEEE Transactions on Wireless Communications, pp. 1-1, 2021.

[6] J. P. Choi and V. W. S. Chan, "Optimum power and beam allocation based on traffic demands and channel conditions over satellite downlinks," IEEE Transactions on Wireless Communications, vol. 4, no. 6, pp. 2983-2993, Nov 2005.

7] U. Park, H. W. Kim, D. S. Oh, and B. J. Ku, "Flexible Bandwidth Allocation Scheme Based on Traffic Demands and Channel Conditions for Multi-Beam Satellite Systems," in IEEE Vehicular Technology Conference, Sep. 2012, pp. 1-5.

[8] T. S. Abdu, E. Lagunas, S. Kisseleff, and S. Chatzinotas, "Carrier and Power Assignment for Flexible Broadband GEO Satellite Communications System,” in 2020 IEEE 31st Annual International Symposium on Personal, Indoor and Mobile Radio Communications, 2020, pp. 1-7.

[9] T. S. Abdu, S. Kisseleff, E. Lagunas, and S. Chatzinotas, "Limits of Smart Radio Resource Assignment in GEO Satellite Communications,' in 2021 IEEE Wireless Communications and Networking Conference (WCNC), 2021, pp. 1-7.
[10] E. Lagunas, M. G. Kibria, H. Al-Hraishawi, N. Maturo, and S. Chatzinotas, "Dealing with Non-Uniform Demands in Flexible GEO Satellites: The Carrier Aggregation Perspective," in 2020 10th Advanced Satellite Multimedia Systems Conference and the 16th Signal Processing for Space Communications Workshop (ASMS/SPSC), 2020, pp. 1-5.

[11] G. Cocco, T. de Cola, M. Angelone, Z. Katona, and S. Erl, "Radio Resource Management Optimization of Flexible Satellite Payloads for DVB-S2 Systems," IEEE Transactions on Broadcasting, vol. 64, no. 2, pp. 266-280, Jun. 2018

[12] A. Paris, I. Del Portillo, B. Cameron, and E. Crawley, "A Genetic Algorithm for Joint Power and Bandwidth Allocation in Multibeam Satellite Systems," in 2019 IEEE Aerospace Conference, March 2019, pp. $1-15$.

[13] N. Pachler, J. J. G. Luis, M. Guerster, E. Crawley, and B. Cameron, "Allocating power and bandwidth in multibeam satellite systems using particle swarm optimization," in 2020 IEEE Aerospace Conference, 2020, pp. 1-11.

[14] S. Kisseleff, B. Shankar, D. Spano, and J.-D. Gayrard, "A new optimization tool for mega-constellation design and its application to trunking systems," in Advances in Communications Satellite Systems. Proceedings of the 37th International Communications Satellite Systems Conference (ICSSC-2019), 2019, pp. 1-15

[15] L. Lei, E. Lagunas, Y. Yuan, M. G. Kibria, S. Chatzinotas, and B. Ottersten, "Beam illumination pattern design in satellite networks: Learning and optimization for efficient beam hopping," IEEE Access, vol. 8, pp. 136655-136667, 2020.

[16] F. G. Ortiz-Gomez, D. Tarchi, R. Martínez, A. Vanelli-Coralli, M. A. Salas-Natera, and S. Landeros-Ayala, "Convolutional Neural Networks for Flexible Payload Management in VHTS Systems," IEEE Systems Journal, pp. 1-12, 2020.

[17] J. Lei and M. A. Vázquez-Castro, "Multibeam satellite frequency/time Duality study and capacity optimization," Journal of Communications and Networks, vol. 13, no. 5, pp. 472-480, Oct 2011.

[18] A. Wang, L. Lei, E. Lagunas, S. Chatzinotas, A. I. P. Neira, and B. Ottersten, "Joint Beam-Hopping Scheduling and Power Allocation in NOMAAssisted Satellite Systems," in 2021 IEEE Wireless Communications and Networking Conference (WCNC), 2021, pp. 1-6.

[19] B. Shankar, M. E. Lagunas, S. Chatzinotas, and B. Ottersten, "Precoding for satellite communications: Why, how and what next?" IEEE Communications Letters, pp. 1-1, 2021.

[20] L. Cottatellucci, M. Debbah, G. Gallinaro, R. Mueller, M. Neri, and R. Rinaldo, Interference Mitigation Techniques for Broadband Satellite Systems. [Online]. Available: https://arc.aiaa.org/doi/abs/10.2514/6.2006-5348

[21] B. Devillers, A. Perez-Neira, and C. Mosquera, "Joint linear precoding and beamforming for the forward link of multi-beam broadband satellite systems," in 2011 IEEE Global Telecommunications Conference GLOBECOM 2011, 2011, pp. 1-6.

[22] V. Joroughi, M. Vázquez, and A. I. Pérez-Neira, "Precoding in multigateway multibeam satellite systems," IEEE Transactions on Wireless Communications, vol. 15, no. 7, pp. 4944-4956, 2016.

[23] — , "Generalized multicast multibeam precoding for satellite communications," IEEE Transactions on Wireless Communications, vol. 16, no. 2, pp. 952-966, 2017.

[24] E. Lagunas, S. Andrenacci, S. Chatzinotas, and B. Ottersten, "Crosslayer forward packet scheduling for emerging precoded broadband multibeam satellite system," in 2018 9th Advanced Satellite Multimedia Systems Conference and the 15th Signal Processing for Space Communications Workshop (ASMS/SPSC), 2018, pp. 1-8.

[25] M. Vázquez, M. R. B. Shankar, C. I. Kourogiorgas, P.-D. Arapoglou, V. Icolari, S. Chatzinotas, A. D. Panagopoulos, and A. I. PérezNeira, "Precoding, scheduling, and link adaptation in mobile interactive multibeam satellite systems," IEEE Journal on Selected Areas in Communications, vol. 36, no. 5, pp. 971-980, 2018.

[26] G. Taricco and A. Ginesi, "Precoding for flexible high throughput satellites: Hot-spot scenario," IEEE Transactions on Broadcasting, vol. 65, no. 1, pp. 65-72, 2019.

[27] M. Grant and S. Boyd, "CVX: Matlab software for disciplined convex programming, version 2.1," http://cvxr.com/cvx, Mar. 2014. 